\title{
Helicopter parameter extraction using joint Time-Frequency and Tomographic Techniques
}

\author{
A Cilliers, WAJ Nel \\ DPSS, CSIR \\ South Africa \\ acilliersecsir.co.za, wajneldcsir.co.za
}

\begin{abstract}
A technique based on time-frequency and tomographic analysis to extract helicopter blade parameters for the purposes of radar non-cooperative target recognition (NCTR) is investigated. The proposed algorithm shows that (under certain conditions) it is feasible to extract the number, blade length, blade tip velocity and angular velocity of a helicopter's main rotor. Simulation results from a point scatterer model is validated through results on measured data from several different commercial helicopters. The developed technique is most suited to pulse-Doppler tracking radar and is shown to be feasible at a range of $11 \mathrm{~km}$ with a radar not specifically designed for this purpose.
\end{abstract}

\section{INTRODUCTION}

The problem of classifying and identifying airborne targets in a combat situation started during the first world war and has become increasingly important with advances in airborne strike power [1]. A branch of engineering theory that attempts to alleviate this problem is radar based NCTR, which are expected to provide useful information at long range in order to obtain a positive identification of possible targets, especially where identify friend or foe techniques are disabled or non-functional. Several different methods are used for the classification and identification process, each concentrating on different aspects of the radar system and of the target.

Most aircraft has some form of rotating structure that causes Doppler modulation of the radar return and can be used to aid in the classification process. Jet engines on a fighter plane or the rotor structure on a helicopter are primary examples. These moving structures causes additional spectral sidebands about the mean target Doppler frequency [1]. Several research efforts have been published on the use of such modulation (also called micro-Doppler effects) for classification purposes which include [2], [3], [4], [5], [6], [7] and [8].

Ref. [9] proposes a method where the (so called L/N) quotient of the blade length and number of blades are estimated by using the Doppler spectrum and the time signal. In realistic scenarios however, the $\mathrm{L} / \mathrm{N}$ technique has limited value due to significant class overlap between different helicopters. In [3] time-frequency analysis of the Doppler spectrum is suggested as an improvement to uniquely determine the number of main rotor blades by counting the number of main rotor blade tip sinusoids in the spectrogram. A spectrogram of recorded data is shown in Figure 1. In cases where the sinusoids are masked by noise or clutter, the analysis can still produce an estimate of blade parity (odd or even number of blades) [5],

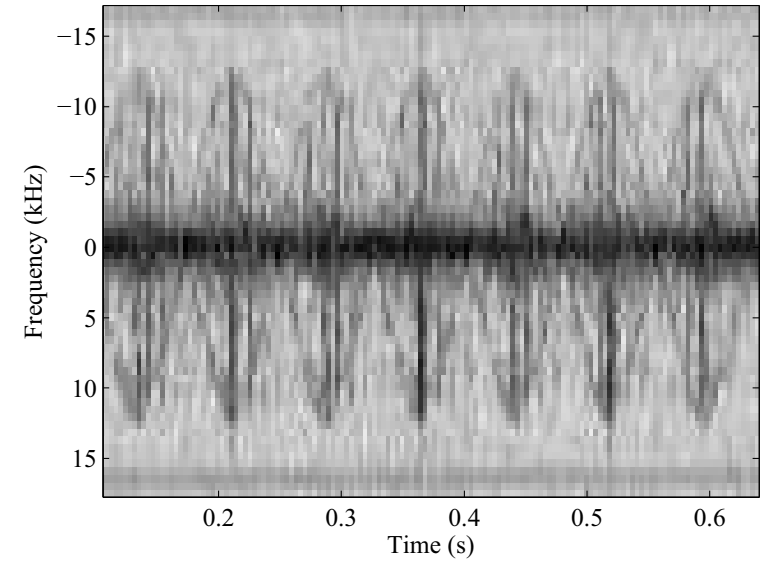

Fig. 1. Spectrogram of recorded helicopter data

since the scattering power of the blade flash is significantly higher than the blade tip scattering. In many radar waveforms however, blade flashes can be missed due to their small angular extent, causing difficulty for automatic analysis approaches. Ref. [10] shows the use of a tomographic imaging technique to create an image of the rotor blades of the helicopter by using a backprojection reconstruction algorithm. The issue of estimating blade parameters such as rotation rate and length using this technique is however not discussed.

The problem of modelling blade returns has also received significant attention. Ref. [11] presents a point-scatterer based model and shows that it is useful to model the general structure of the micro-Doppler effects. An analysis of modelled and measured data using high-resolution time-frequency transforms is presented. The work by [6] and [12] shows how the EM backscattering properties of the helicopter blade can be calculated using physical optics and quasi-stationary methods. Both these papers give the time-varying as well as timefrequency analysis of recorded rotor data and [6] investigates both horizontal and vertical polarisations.

This paper aims to extend the field of Helicopter Blade Modulation (HBM) analysis by showing how the work in [10] and [11] can be combined to form a new technique for accurately estimating the rotor tip velocity, whilst easily and unambiguously providing information regarding the number of blades and the blade length. The paper shows simulated results as well as a summary of actual measured data on several civilian helicopters which show that the technique is 
both accurate and feasible in real tracking radar scenarios, where reasonably long dwell times are available.

\section{Modelling}

To model the micro-Doppler effects of the HBM, a point scatterer model similar to that in [11] can be used. Careful placement of the scatterers at better than a $\frac{1}{4} \lambda$ will ensure that the blade flash structure is preserved and that no grating lobes result in the angular scattering pattern of the blades. This model also generates blade tip scattering. Scatterers are placed at a start radius of $L_{\text {min }}$ from the hub centre, and have a maximum radial distance of $L_{\max }$. The return from the helicopter body can either be simulated by a single large amplitude scatterer at the centre position, or can be ignored, if not deemed pertinent to the question being answered by the model. To calculate the resultant amplitude and phase of the blades the following equation can be used

$$
S_{r e t}=\sum_{k=1}^{K} \alpha_{k} e^{-j 4 \pi R_{k} / \lambda}
$$

where $\alpha$ is a chosen constant amplitude for each scatterer on the blade, $\lambda$ is the wavelength and $R_{k}$ is a function of the position of the main rotor blade relative to the radar at the instant of measurement, the minimum and maximum blade length $\left(L_{\min }\right.$ and $\left.L_{\max }\right)$ and the spacing of the scatterers on the blade. To calculate the radar return, it is assumed that the blade is stationary during the measurement instant and a new blade position is calculated for each blade at each measurement instant. $\mathrm{K}$ is the total number of scatterers used to represent the blades and there are $\mathrm{N}$ blades in the simulation.

In a similar fashion the model can be extended to include the effect of the tail rotor when it is visible, but the processing techniques used later in this paper are not affected much by the presence of Doppler returns from the tail rotor.

The spectrogram of the data is obtained by taking a Mpoint Blackman windowed FFT of $S_{\text {ret }}$. For the case shown in Figure 2, M is chosen as 64 samples and there are no overlap between adjacent FFTs. $L_{\min }$ and $L_{\max }$ were chosen as $0.2 \mathrm{~m}$ and $5.35 \mathrm{~m}$ respectively and a total of 1752 scatterers were used to represent the three bladed main rotor. The model rotates with an RPM of 390 and the radar PRF is simulated as 35 $\mathrm{kHz}$.

Comparing Figure 2 and Figure 1 it is clear that the scattering model produces micro-Doppler effects similar to those observed in measured radar data (bar the effects of the tail rotor and main body return). When a main rotor blade is perpendicular to the radar line of sight a blade flash occurs that contains Doppler content dependent on $L_{\min }, L_{\max }$ and the angular rate, $\omega$ of the main rotor as well as the radar wavelength. By analysing the relative position (in time) of the coming and going blade flashes the parity of $\mathrm{N}$ (odd or even) can be determined. In this case 3 unique sinusoids are observed, indicating 3 main rotor blade tips as described in [3].

As the blade rotates from the perpendicular the received energy reduces and the Doppler frequency of the blade tip

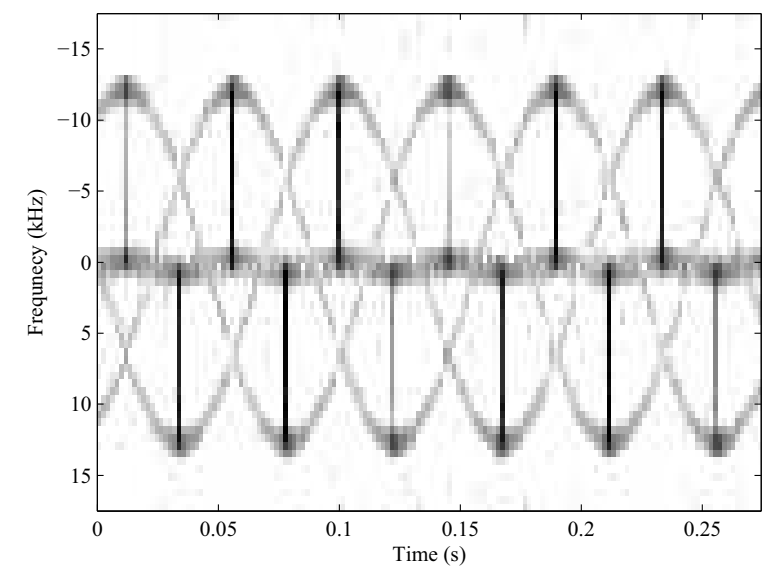

Fig. 2. Spectrogram of simulated data. Three bladed helicopter with blade length of $5.35 \mathrm{~m}$ and a RPM of 390 .

reduces up to the point where the returned signal from the blade tip has zero Doppler return when the blade is parallel to the radar line of sight.

The Doppler frequency of a moving scatterer can be written as

$$
f_{D}=\frac{2 v}{\lambda}
$$

The maximum Doppler bandwidth represented by the radar data adheres to the Nyquist criterion for complex sampling and is thus equal to the radar pulse repetition frequency (PRF). To ensure unambiguous sampling of the data the radar PRF has to be chosen as

$$
P R F \geq \frac{4 \omega L_{\max }}{\lambda}
$$

where $\omega$ is the angular rate of the main rotor blade.

For the simulated data in this paper the wavelength was chosen as X-band to coincide with the radar being used to record data and the waveform PRF was chosen to unambiguously sample the largest expected Doppler bandwidth.

The simulation does not mathematically account for the signal to noise dependence on range as would be the case in measured data nor does it properly account for the angular dependence of the blade tip scattering since the scattering model is based on omni-directional scatterers. Even with these constraints the model is however useful to investigate techniques to extract information from the blade tip and blade flash scattering mechanisms.

\section{BLADE PARAMETER EXTRACTION}

This section describes the signal processing algorithm that can be used to extract blade parameters from both simulated and recorded data. The block diagram in Figure 3 gives an overview of the key processing steps of the algorithm. The first step is to generate or load the data. Section II describes the simulation data generation and the real data was recorded during a helicopter trial and is described and discussed in Section IV-A.

Since a moving helicopter generates a mean Doppler response at the velocity of the helicopter the first step in 


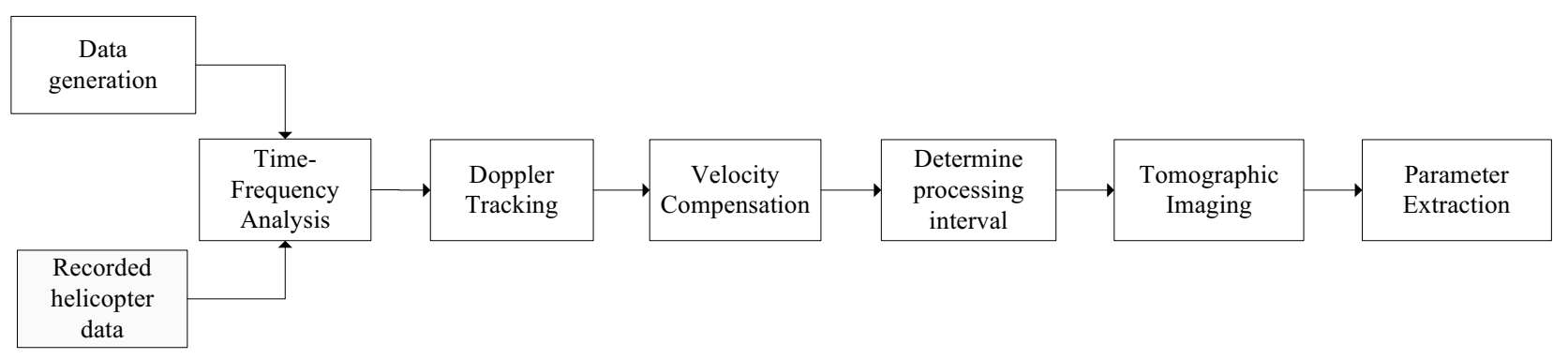

Fig. 3. Block diagram of the HBM parameter extraction algorithm.

the algorithm is to correct for this response. This is done by generating the spectrogram of the data as discussed in the previous section and then performing Doppler centroid tracking on the data. For each spectral estimate (FFT output) a phase correction factor is calculated by which the time-domain data have to be corrected to remove the target mean Doppler. This step is known as velocity compensation and results (after another FFT) in the Doppler spectrogram of the data with the target at zero Doppler. Ideally the velocity compensation step should result in a peak Doppler line for the helicopter's fuselage that does not drift over multiple Doppler bins for the chosen processing interval. With this in mind a processing interval can be chosen that would be a good input for the tomographic processing to follow. The processing interval length should be chosen such that at least one and preferably more than one full blade revolution is visible in the data. The last step in the processing algorithm is to use tomographic imaging to form an image of the rotor blade tips and use this to extract information regarding the RPM, length and number of blades.

\section{A. The Radon and Inverse Radon Transform}

To calculate a set of 1-D projections of a 2-D function $f(x, y)$ as $P(\alpha, r)$ the Radon transform as defined in Equation 4 can be used [13].

$$
P(\alpha, r)=\iint f(x, y) \delta(x \cos \alpha+y \sin \alpha-r) d x d y
$$

Here $\alpha$ is the angle of the line $v$ (see Figure 4), onto which the projection is made and $r$ indicates the distance from the origin at which the line integral for a specific point of the projection is carried out. $P(\alpha, r)$ is a complete collection of 1-D projections of $f(x, y)$.

The inverse of this theory is useful in the case where a set of projections of a 2-D function at various angles are known and it is required to reconstruct the function from these projections.

The Fourier slice theorem states that the 1-D Fourier transform of the projections of a function onto a line $v$ at a specific angle $\alpha$ is equal to a slice of the 2-D Fourier transform of the function $f(x, y)$ at the same angle (see [14]). This theorem can therefore be used to generate a reconstruction of the function $f(x, y)$ given a number of 1-D projections. To do this, the 2-D Fourier transform of the function is first constructed from the projections and their known projection angles and then an

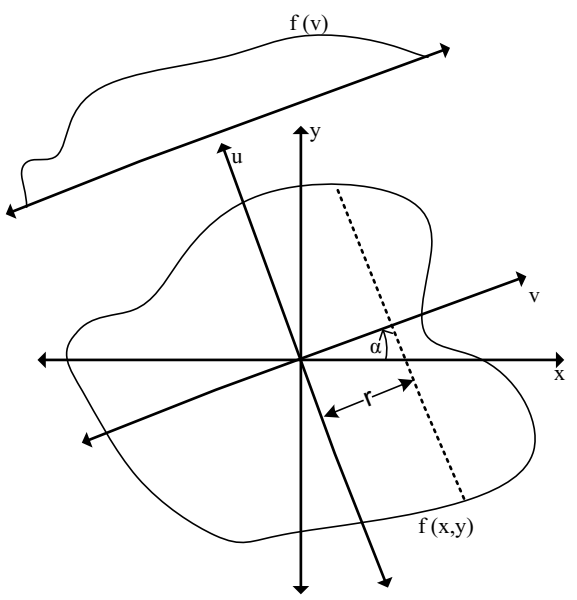

Fig. 4. Projection of object

Inverse 2-D Fourier transform is used to calculate the original 2-D function.

This process is also known as the Inverse Radon Transform (IRT) and can be described mathematically by Equation 5 .

$$
f(x, y)=\int_{-\infty}^{\infty} \int_{-\infty}^{\infty} S_{\alpha}(t) e^{j 2 \pi(u x+v y)} d u d v
$$

\section{B. Helicopter blade parameter extraction}

Assuming that the helicopter main rotor is horizontal, that the helicopter is stationary at a range of $R_{0}$ from the radar, that $R_{0}>>L$ and that the elevation angle is close to zero, the range of the blade tip of a helicopter to the radar can be derived as

$$
\begin{aligned}
& R(t)=R_{0}^{2}+L^{2}+2 R_{0} L \cos (\alpha(t)) \cos (\beta) \\
& R(t) \cong R_{0}+L \cos (\alpha(t))
\end{aligned}
$$

where $\alpha(t)$ is the angle to the blade tip vs time $t$ and $\beta$ is the elevation angle of the helicopter main rotor from the horizon relative to the radar.

The Doppler frequency of the blade tip as a function of time can now be written as

$$
\begin{aligned}
f_{d}(t) & =2 v(t) / \lambda \\
& =-\frac{2 L \omega}{\lambda} \sin (\alpha(t))
\end{aligned}
$$

where $\omega$ is the main rotor rate of turn in $\mathrm{rad} / \mathrm{s}$. Equation 8 shows that the Doppler frequency of the blade tip is a 

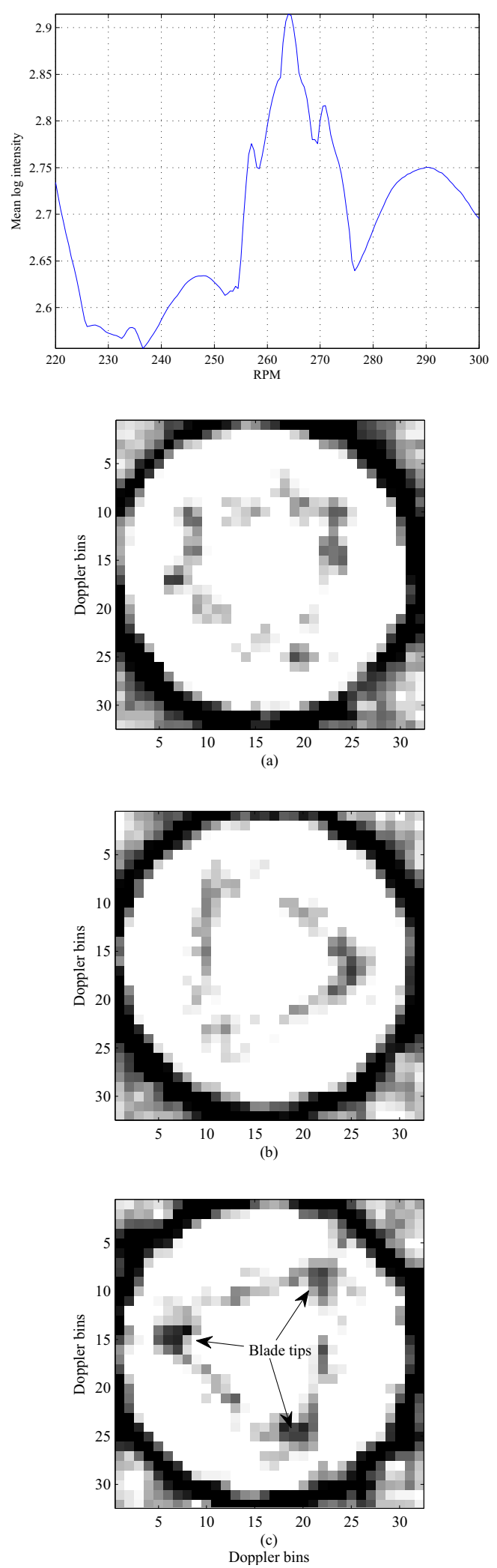

Fig. 5. Inverse Radon Transform for simulated data at different angular rate (a) $\mathrm{RPM}=235$, (b) $\mathrm{RPM}=285$ and (c) $\mathrm{RPM}=265$ projection of the cross-range coordinate of the blade tip barring a scaling factor of $2 \omega / \lambda$ and that a series of such projections is formed by the spectrogram of the Doppler data. If the helicopter is not stationary these projections will be situated relative to the Doppler of the body of the helicopter, requiring velocity compensation to remove this main Doppler component.

The 2-D image of the blade tips can now be formed using the IRT if the angle of projection for each profile is known. Assuming $\omega$ is a constant, the angle of each projection can be estimated as a linear function vs time with unknown slope.

A linear search method can be used to estimate the correct angular rate by using the IRT image focus as the fitness criteria. The search boundaries can either be obtained by estimating the period from the sinusoids in the spectrogram and performing a search around this point, or by using knowledge of the different RPMs of known helicopters, which ranges from 200 - 600 RPM. The IRT process is then applied to the data at a linearly spaced set of rates throughout this search space. The most likely angular rate can then be estimated as that rate at which the image produced by the IRT has the best focus. In the results presented here, the most focused image was simply chosen as that image which produced the highest peak amplitude for the blade tips in the sequence of IRT images.

To illustrate how the IRT is applied for this problem, Figure 5 shows the projections at three different angular rates for simulated data, where the RPM of the main rotor is simulated as 265. From these graphs the search process for the correct angular rate, $\omega$ can be seen. The blade tips will only focus when the correct value is used as seen in (c). (a) and (b) show two cases where the angular rate is approaching the correct value. For (c) the image focuses the blade tips, and from there the correct RPM can be determined. The first graph in Figure 5 shows the mean log intensity of each image produced by an IRT for different the RPM's in the linear search. It is clear that the peak value corresponds closely with the correct RPM of 265 .

From Equation 2 the blade tip velocity is determined by an estimate of the blade tip Doppler frequency as obtained from the Doppler-Doppler image produced by the IRT. To calculate this an estimate of the coordinates of the pixel with maximum intensity in the Doppler-Doppler image is made as $f_{D}(x, y)$, where $x$ and $y$ is an estimate of the Doppler frequency on each axis of the image. Using Euclidean distance from the centre of the image the blade tip Doppler frequency can be estimated as

$$
f_{D_{\text {tip }}}=\sqrt{x_{\text {tip }}^{2}+y_{\text {tip }}^{2}}
$$

From the estimate of the RPM and of the blade tip velocity the blade length can now be calculated using Equation 10.

$$
\begin{aligned}
R P M & =\frac{\omega}{2 \pi} 60 \\
L & =\frac{v}{\omega}
\end{aligned}
$$

It can be argued that the RPM can be determined directly 


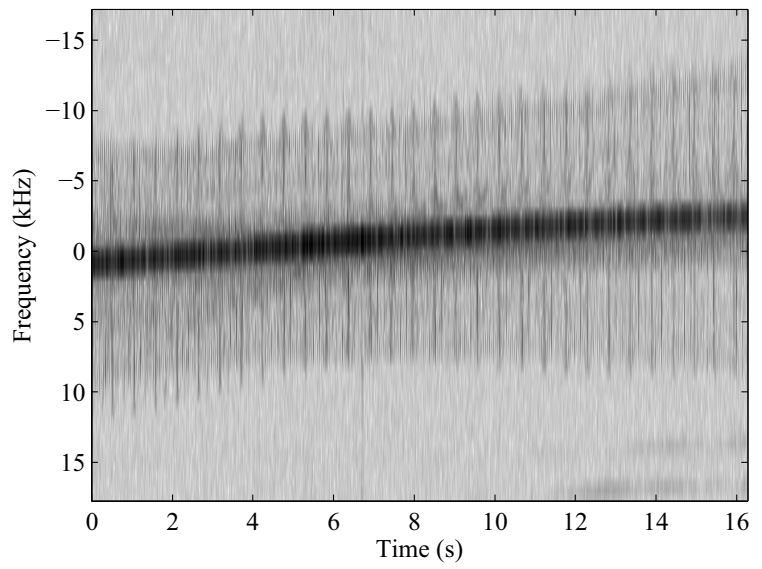

Fig. 6. Spectrogram of recorded data for an AS350B helicopter before velocity compensation.

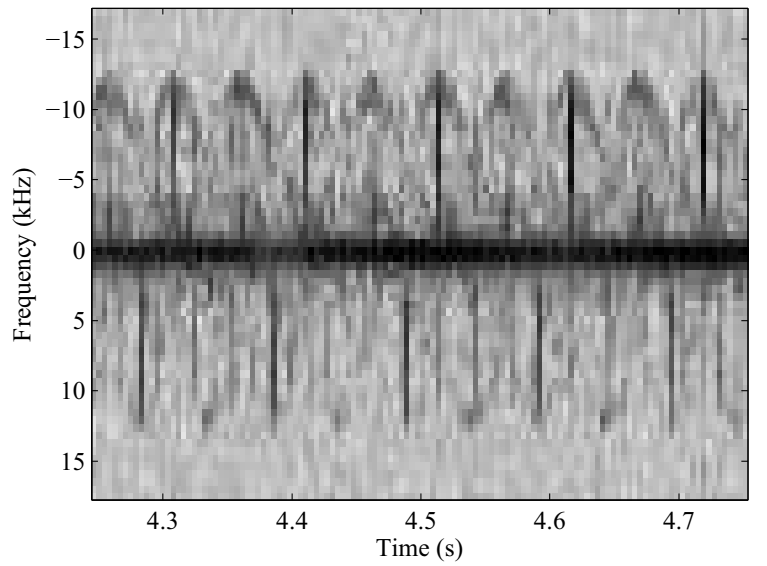

Fig. 7. Spectrogram of recorded data for an AS350B after velocity compensation.

from the spectrogram of the data, by either using the sinusoids and the zero crossings, or using an estimate of the maximum Doppler of the blade flash and an estimate of the period of the sinusoids. However the zero crossings in the measured data is obscured by the Doppler return of the helicopter body, and it is difficult to estimate the sinusoid period in low SNR conditions. The value of the IRT lies in the fact that it produces a noncoherent processing gain which increases the SNR with which estimates are made. Another advantage of the IRT technique is that it produces an accurate estimate of the rotor RPM in the process.

\section{TRIAL DATA}

\section{A. Trial}

Careful thought went into the selection of the different helicopters that were used for the trial. The aim was to choose helicopters that were in different classes, as well as helicopters of the same class, where a class is determined by the number of main rotor blades. Another consideration was to choose helicopters ranging in blade length from being very similar to being significantly different within each class. This ensured

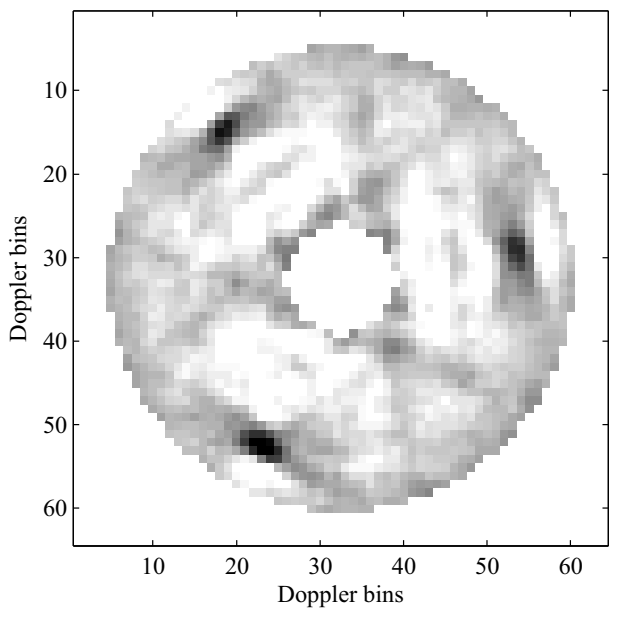

Fig. 8. Tomographic results of recorded helicopter data

that the data will be useful for a separability analysis in the future. Table I shows the ground truth rotor parameters of the various helicopters that were used in the trial. This data was taken from a database of about a 100 helicopters the parameters of which were extracted from several sources.

\section{B. Results}

At present a detailed analysis of all the recorded trial data and the performance of the proposed algorithm is not yet available. The data presented in this section does however show that the technique is feasible for use on measured data.

The spectrogram of the measured data before the velocity compensation for the AS $350 \mathrm{~B}$ is shown in Figure 6. Figures 7 and 8 show the spectrogram after velocity compensation and IRT for the measured radar data. The parity and sinusoids can be seen in the time-frequency plot from where the estimate of the angular rate was determined. Note how clearly the three blade tips are visible in the image formed by the IRT, even though the amplitude of the coming and going blade tips are clearly not equal in Figure 7. For this case the estimated RPM of the helicopter main rotor was calculated as 392 which constituted a $0.04 \%$ error compared to that of the value in the database. Similar results for other helicopters at different ranges is shown in Table II. It is clear that even at ranges as far as $11 \mathrm{~km}$ the technique produces feasible results.

In the near future the algorithm will be characterised and qualified using this dataset. The dataset itself contains examples of different flight profiles for both inbound, outbound, hovering, circling and even ascending and descending helicopters. This dataset will be useful to analyse the sensitivity of the various extracted parameters for these different profiles and provide understanding regarding the separability of the different helicopters. This will provide useful insight towards an analysis of the required parameters that has to be estimated in order to identify helicopter make and model uniquely.

\section{RADAR SYSTEM CONSIDERATIONS}

The helicopter data was recorded by an experimental Xband monopulse tracking radar where the waveforms were 
TABLE I

HELICOPTERS USED FOR THE TRIAL

\begin{tabular}{|c|c|c|c|c|c|c|c|c|}
\hline \multicolumn{9}{|c|}{ Class type } \\
\hline \multicolumn{3}{|c|}{2 Main Rotor Blades } & \multicolumn{3}{|c|}{3 Main Rotor Blades } & \multicolumn{3}{|c|}{4 Main Rotor Blades } \\
\hline Helicopter & $\mathbf{L}(\mathbf{m})$ & RPM & Helicopter & $\mathbf{L}(\mathbf{m})$ & RPM & Helicopter & $\mathbf{L}(\mathbf{m})$ & RPM \\
\hline Bell 206 (Jet Ranger) & 5.08 & 380 & Colibri EC 120 & 5 & 414 & Bell 407 & 5.34 & 413 \\
\hline Bell 206 (Long Ranger) & 5.64 & 394 & AS 350B Squirrel & 5.35 & 390 & & & \\
\hline Robinson R22 & 3.85 & 491 & Aloutte II & 5.1 & - & & & \\
\hline Robinson R44 & 5.05 & 400 & Eurocopter EC 130 & 5.34 & - & & & \\
\hline
\end{tabular}

TABLE II

RECORDED DATA RESULTS

\begin{tabular}{|c|c|c|c||c|c||c|c|c|c||c|}
\hline \multicolumn{4}{|c||}{ Helicopter Parameters } & \multicolumn{3}{c||}{ Experiment Parameters } & \multicolumn{3}{c||}{ Estimated Parameters } & \% Error \\
\hline Type & N & L $(\mathbf{m})$ & RPM & Range $\mathbf{( k m )}$ & Profile & N & L $(\mathbf{m})$ & RPM & L (m) & RPM \\
\hline Bell 206 JR & 2 & 5.08 & 392 & 3 & Outbound & 2 & 5.07 & 389 & $0.2 \%$ & $0.77 \%$ \\
\hline Bell 206 LR & 2 & 5.64 & 394 & 3.5 & Descent & 2 & 5.52 & 393.5 & $2.12 \%$ & $0.12 \%$ \\
\hline AS 350 B & 3 & 5.35 & 390 & 11.4 & Inbound & 3 & 5.25 & 392 & $1.8 \%$ & $0.04 \%$ \\
\cline { 3 - 11 } & & & & 10 & Outbound & 3 & 5.16 & 391 & $3.5 \%$ & $0.51 \%$ \\
\hline R22 & 2 & 3.85 & 491 & 1.7 & Circling hover & 2 & 3.83 & 529 & $1 \%$ & $21 \%$ \\
\hline Bell 407 & 4 & 5.34 & 413 & 2.75 & Circling hover & 4 & 5.1 & 413.1 & $4.5 \%$ & $0.01 \%$ \\
\hline
\end{tabular}

designed to have an unambiguous PRF. The specific radar used measures only vertical polarisation. It is believed that better results can be achieved when using a horizontally polarised radar operating in a lower frequency band as discussed in [6] and [15].

\section{CONCLUSION}

The paper proposed a new method using tomographic techniques to extract pertinent helicopter blade parameters which aids in the process of identifying helicopter targets. Both the simulation and measured results show that the technique seems both feasible and effective for tracking radar NCTR purposes and that the RPM estimation with the tomographic technique appears to be very accurate. The knowledge of the rotor RPM also enables estimation of the number of blades and blade length which have been identified as problematic in previous work as discussed in the Introduction.

Future work will include the detailed analysis of the measured radar data to investigate the performance of the algorithm with varying target ranges, different flight profiles and to investigate the interclass separability. The influence of SNR and the use of the tail rotor modulation will also be investigated. The simulation model will also be used to further investigate the effect of different radar frequencies as well as the effect of polarisation. The effect of double main rotor helicopters will also be investigated.

\section{ACKNOWLEDGMENT}

The authors would like to thank all the members of the DPSS Radar and EW group who were involved in the Helicopter trials, as well as Armscor for the funding of the project.

\section{REFERENCES}

[1] P. Tait, Introduction to Radar Target Recognition. United Kingdom: The Institution of Electrical Engineers, 2005.
[2] V. Chen, F. Li, S. Ho, and H. Wechsler, "Analysis of micro-doppler signatures," Radar, Sonar and Navigation, IEE Proceedings -, vol. 150, no. 4, pp. 271-6-, 1 Aug. 2003.

[3] S. Yoon, B. Kim, and Y. Kim, "Helicopter classification using timefrequency analysis," Electronics Letters, vol. 36, no. 22, pp. 1871-1872, 26 Oct 2000.

[4] J. Martin and B. Mulgrew, "Analysis of the theoretical radar return signal form aircraft propeller blades," Radar Conference, 1990., Record of the IEEE 1990 International, pp. 569-572, 7-10 May 1990.

[5] B. Bullard and P. Dowdy, "Pulse doppler signature of a rotary-wing aircraft," Aerospace and Electronic Systems Magazine, IEEE, vol. 6, no. 5, pp. 28-30, May 1991.

[6] P. Pouliguen, L. Lucas, F. Muller, S. Quete, and C. Terret, "Calculation and analysis of electromagnetic scattering by helicopter rotating blades," Antennas and Propagation, IEEE Transactions on, vol. 50, no. 10, pp. 1396-1408, Oct 2002.

[7] M. Bell and R. Grubbs, "Jem modeling and measurement for radar target identification," Aerospace and Electronic Systems, IEEE Transactions on, vol. 29, no. 1, pp. 73-87, Jan 1993.

[8] J. Misiurewicz, K. Kulpa, and Z. Czekala, "Analysis of recorded helicopter echo," 14-16 Oct 1997, pp. 449-453.

[9] C. Rotander and H. V. Sydow, "Classification of helicopters by the $1 / \mathrm{n}$ quotient," Radar 97 (Conf. Publ. No. 449), pp. 629-633, 14-16 Oct 1997.

[10] G. Fliss, "Tomographic radar imaging of rotating structures," in Synthetic aperture radar; Proceedings of the Meeting, Los Angeles, CA, Jan. 20, 21, 1992 (A93-32508 12-32), p. 199-207., ser. Presented at the Society of Photo-Optical Instrumentation Engineers (SPIE) Conference, R. D. McCoy and M. E. Tanenhaus, Eds., vol. 1630, May 1992, pp. 199-207.

[11] V. Chen, F. Li, S. Ho, and H. Wechsler, "Micro-doppler effect in radar: phenomenon, model, and simulation study," Aerospace and Electronic Systems, IEEE Transactions on, vol. 42, no. 1, pp. 2-21, Jan. 2006.

[12] S. Yang, S. Yeh, S. Bor, S. Huang, and C. Hwang, "Electromagnetic backscattering from aircraft propeller blades," Magnetics, IEEE Transactions on, vol. 33, no. 2, pp. 1432-1435, Mar 1997.

[13] F. Zhang, G. Bi, and Y. Chen, "Tomography time-frequency transform," Signal Processing, IEEE Transactions on [see also Acoustics, Speech, and Signal Processing, IEEE Transactions on], vol. 50, no. 6, pp. 12891297, Jun 2002.

[14] S. Qureshi, S. Mirza, and M. Arif, "Inverse radon transform-based image reconstruction using various frequency domain filters in parallel beam transmission tomography," Engineering Sciences and Technology, 2005. SCONEST 2005. Student Conference on, pp. 1-8, 27-27 Aug. 2005.

[15] H. Green, "Electromagnetic backscattering from a helicopter rotor in the decametric wave band regime," Antennas and Propagation, IEEE Transactions on, vol. 42, no. 4, pp. 501-509, Apr 1994. 